\title{
Assessment of quality of life in bronchial asthma patients
}

Introduction: Asthma is a common chronic disease that affects persons of all ages. People with asthma report impact on the physical, psychological and social domains of quality of life. Health-related quality of life (HROLL) measures have been developed to complement traditional health measures such as prevalence, mortality and hospitalization as indicators of the impact of disease. Objective and Study Design: The objective of this study was to assess HRQoL in Bronchial asthma patients and to relate the severity of asthma with their quality of life. About 85 asthma patients were evaluated for HROoL and their pulmonary function tests values were correlated with HRQoL scores. Results and Conclusion: It was found that asthma patients had poor quality of life. There was greater impairment in quality of life in females, obese and middle age patients indicating that sex, body mass index and age are determinants of HRQOL in asthma patients.

Key words: Bronchial asthma, health-related quality of life, pulmonary function tests

\section{N. Nalina,} M. R. Sathish Chandra', Umashankar

Department of Physiology, Kempegowda Institute of Medical

Sciences, 'Department of Community Medicine, BGS Global Institute of Medical Sciences,

Bengaluru, Karnataka, India

Address for the Correspondence: Dr. N. Nalina,

Department of Physiology, Kempegowda Institute of Medical Sciences, Near BDA Complex,

Bhanashankari II Stage, Bengaluru - 560 070, Karnataka, India.

E-mail: dr.nalinarani@gmail.com

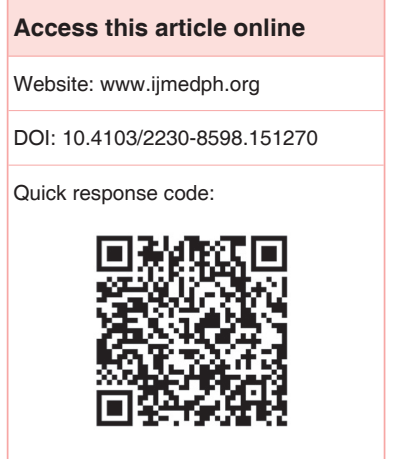

\section{INTRODUCTION}

Asthma is one of the most common chronic diseases in the world. Asthma is a problem worldwide, with an estimated 300 million affected individuals. ${ }^{[1]}$ Despite hundreds of reports on the prevalence of asthma in widely differing populations, the lack of a precise and universally accepted definition of asthma makes reliable comparison of reported prevalence from different parts of the world problematic. In 1989, the Global Initiative for Asthma (GINA) program was initiated in an effort to raise awareness among public health and government officials, health care workers, and the general public that asthma was on the increase. ${ }^{[1]}$

In the global burden of asthma report of the GINA, the prevalence of asthma in different countries has been considered to range from $1 \%$ to $18 \%$ of the population. ${ }^{[1]}$ Both morbidity and mortality from asthma are significant. Social and economic factors are necessary for understanding asthma and its care, whether viewed from the perspective of the individual sufferer or the health care delivery system. ${ }^{[2]}$ The number of disability-adjusted life years lost due to asthma worldwide is similar to that for diabetes, liver cirrhosis and schizophrenia. Absence from days lost from work are reported as substantial social and economic consequences of asthma in studies from the Asia-Pacific region, India, Latin America, United Kingdom, and United States. ${ }^{[1,2]}$

In patients with a known diagnosis of asthma, respiratory symptoms are important determinants of reduced health-related quality of life (HRQoL). ${ }^{[3]}$ In developing countries, chronic respiratory diseases represent a challenge to public health because of their increasing frequency and severity and the projected trends and economic impact. It is estimated that asthma accounts for about 2,50,000 deaths per year worldwide. Historically, studies of patient outcomes in asthma have focused on clinical and physiologic measures. More recently, however, there is growing recognition that such clinical measures do not provide a complete, or in some cases, accurate, view of the impact of a disease on an individual's physical, social, or emotional well-being.

Health-related quality of life has been considered an important variable to be managed in airway diseases. ${ }^{[4]}$ Asthma can reduce HRQoL as a result of profound physical and psychosocial complications. Besides physical symptoms, asthma patients may exhibit fatigue, psychomotor sluggishness, irritability, and mood and cognitive disturbances. This combination of physical, emotional, and functional problems may diminish HRQoL. ${ }^{[5]}$ 
Evaluation of HRQoL provides a primary outcome measure in patients with chronic diseases. Asthma is very common condition that is recognized as the cause of reduced quality of life. Asthmatic symptoms lead to impairment in the physical, emotional, and social aspects of a patient's life. ${ }^{[5]}$ The assessment of HRQoL is thus rendered even more relevant in patients who have chronic diseases such as asthma which can only be treated, but not healed, as the achievement of the best possible quality of life becomes the paramount objective in the management of the patient.

In response, HRQoL measures are increasingly being integrated into clinical research in asthma. The goals of asthma therapy are to improve the patients' quality of life by preventing chronic and troublesome symptoms, maintaining "normal" lung function, maintaining normal activity levels, preventing recurrent exacerbations and providing optimal pharmacotherapy with minimal adverse effects. ${ }^{[6]}$ The issue of the quality of life of patients with bronchial asthma is very often overlooked in the clinical practice.

Therefore, this study was conducted with the objective to assess the effect of bronchial asthma and its severity on the HRQoL.

\section{MATERIALS AND METHODS}

All patients with mild to moderate persistent asthma (GINA guidelines) aged between 18 and 65 years attending chest and tuberculosis (TB) department, Kempegowda Institute of Medical Sciences, Bangalore during June 2012 to December 2012 were included in the study based on inclusion and exclusion criteria. The patients with clinically stable with no exacerbation or hospital admission and no change in medication dosage or frequency in the last 4 weeks in order to avoid any bias in patients' opinion about their health status. The study was approved by the Institutional ethical committee. Patients with severe persistent asthma and severe chronic obstructive pulmonary disease or with $\mathrm{h} / \mathrm{o}$ severe respiratory tract infection in past 4 weeks were excluded from the study. Patients with h/o chronic rhino-sinusitis, gastroesophageal reflux, recurrent viral lower respiratory tract infections, TB, chronic bronchitis or emphysema were also excluded from the study.

\section{Methodology}

Sociodemographic characteristics such as age, education, family income, smoking habit, occupation etc., and history of other allergic disease were collected from patients with mild to a moderate persistent Asthma. Their height and weight were measured, and body mass index (BMI) was calculated. The resting parameters such as pulse rate, blood pressure, and body temperature were recorded. These patients were subjected for pulmonary function tests (PFT) after obtaining informed consent. PFT were done with RMS Helios 501 Spirometer to assess the severity of asthma. Baseline lung function like forced expiratory volume in $1 \mathrm{~s}$ (FEV1), forced vital capacity (FVC), FEV1/FVC, peak expiratory flow (PEF), and forced expiratory flow (FEF) 25-75/FVC were determined. ${ }^{[7]}$

Then, they were assessed for HRQoL using Asthma Quality of Life Questionnaire (AQLQ). Each participant completed the self- administered questionnaire. The Asthma HRQoL questionnaire ${ }^{[5]}$ is a disease-specific 32-item instrument, including 4 domains: Symptoms, emotions, exposure to environmental stimuli and activity limitations. ${ }^{[5,8]}$ Patients rated the impairments they have experienced during the previous 14 days and responded to each item on 7-point scales. The scoring was done by dividing the 32 items in questionnaire into 4 domains where items: 1-5, 11, 19, 25, 28, 31, 32 was included under Activity limitation domain. Items: 6, 8, 10, 12, 14, 16, 18, 20, 22, 24, 29, 30 was included for calculating Symptoms domain. For Emotional function domain items 7, 13, 15, 21 and 27 was included. The Environmental stimuli domain was calculated using items 9, 17, 23 and 26. Individual items were equally weighted and mean AQLQ(S) score was obtained by adding all 32 responses and dividing it by 32 . The scores for 4 domains were analyzed by adding the response of each of items in the domain and dividing by number of items in particular domain. Thus, the overall scores and domain scores range from 1 to 7 with higher scores indicating better quality of life. Score of 7 by the patient was considered has no impairments due to their asthma and score 1 at the other end of the range was severe impairments. The score 4 in the middle of the range indicated moderate degree of impairment. Thus the degree of impairments the patient was experiencing due to their asthma was evaluated by the scoring and as score drops below 7 the level of impairment and thus quality of life was interpreted. The quality of life scores was correlated with FEV1, FVC, FEV1/FVC, PEF, FEF25-75/FVC values and effect of severity of asthma on quality of life was evaluated.

\section{Statistical analysis}

The Statistical software SPSS 17.0 (SPSS version 17.5. IBM, Armonk, USA.) was used for the analysis of the data and Microsoft word and Excel (Microsoft corporation, USA.) have been used to generate tables. The PFT and quality of life parameters were described by means and standard deviations (SD) for different age groups, gender and BMI The correlation was calculated to assess the relationship between AQLQ(S) scores and asthma severity.

\section{RESULTS}

Based on inclusion and exclusion criteria 85 bronchial asthma patients were included in the study, of which 44 were females and 41 were males. The age group included were $18-65$ years and the mean (SD) was $35 \pm 11$ years for both males and females [Table 1]. The patients were grouped based on their age and BMI.

The PFT results are given in Tables 1 and 2 for different age groups, in males and females and patients grouped based on BMI. The mean FEV1 (\% predicted) values were greater in patients $<30$ years compared with the other age groups. The FEV1 values were greater in patients with BMI of 18.5-24.9 compared with patients with BMI $<18.5$ and 25-29.9 but lesser than patients with BMI > 30 [Table 1].

\section{Gender and quality of life scores}

For the total patient population, the mean of total AQLQ score was $4.12 \pm 1.09$ and mean of subscores were $4.01 \pm 1.2$ for symptom 


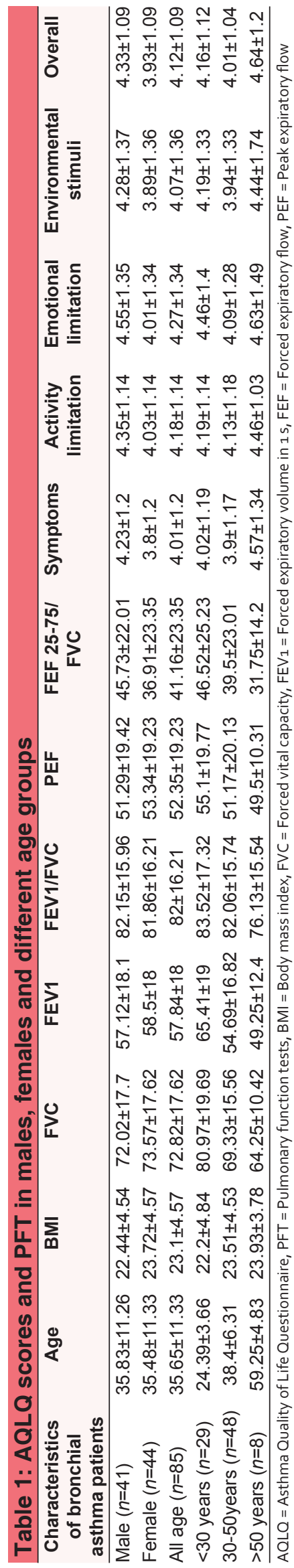

domain, $4.18 \pm 1.14$ for activity limitation domain, $4.27 \pm 1.34$ for emotional function domain and $4.07 \pm 1.36$ for environmental stimuli domain. The mean of total AQLQ score and subscores of all domains were greater in male patients compared to female patients [Table 1].

Age and Quality of life scores: The mean of total AQLQ score and mean of subscores of all domain was greater in patients $<30$ years compared to the scores of patients between 30 and 50 years. The mean of scores for patients $>50$ years was greater when compared with other age groups [Table 1].

Body mass index and quality of life scores: The mean of total AQLQ scores was better in patients with normal BMI of 18.5-24.9 compared to the other groups. The AQLQ scores for all domains was greater in patients of BMI 25-29.9 than in patients with BMI $<18.5$ but lesser than the patients of normal BMI in symptom and activity limitation domain. The scores were least for all domains in patients with BMI > 30 except emotional function domains [Table 2].

\section{Correlation between pulmonary function tests and quality of life scores}

There was a positive correlation between AQLQ scores and PFT scores with greater severity associated with lower AQLQ [Table 3].

\section{DISCUSSION}

Bronchial Asthma is one of the major causes of morbidity and has a major impact on quality of life of the patients. In the present study, HRQoL was impaired in patients with Bronchial Asthma. There was maximum limitation in Symptoms domain of HRQoL compared to other 3 domains. In female patients, HRQoL was more limited compared to male patients and limitation was more for Symptoms domain. There was an impairment in HRQoL in underweight (BMI $<18.5)$ and overweight $(\mathrm{BMI}>25)$ patients compared to patients with normal BMI. In obese $(\mathrm{BMI}>30)$ patients the impairment in HRQoL was more severe compared to other groups except in Emotional function domain. In overweight and underweight patients the Symptoms domain was more impaired unlike obese patients where impairment was more severe in Environmental stimuli domain. The HRQoL was less impaired in asthma patients $<30$ years than patients between 30 and 50 years, but more impaired than patients $>50$ years.

A positive correlation was observed between HRQoL and disease severity in our study (greater adverse impact of asthma). Patients with impaired PFT had more limitation in HRQoL and also in all 4 domains.

Kalpaklioğlu and Baççioğlu has seen that gender and age has an impact on HRQoL in patient with asthma..$^{[9]}$ The findings in the present study are in agreement with the previous studies that have concluded that the determinants of impaired quality of life in Asthma patients were age and sex. $\cdot^{[10-12]}$ Naleway et al. have found that women have poorer outcomes for asthma than men regarding 
HRQoL and symptoms, and our study supports this. ${ }^{[13]}$ For women with asthma, fluctuations in endogenous hormone levels are correlated with changes in their asthmatic condition. In Indian setting, the women have major role to play both in the society and at home thus the morbid disease like asthma has an additive role in an impairment of HRQoL. The stress faced by Indian women may aggravate the asthma condition thus leading to impaired HRQoL.

In the study carried out by Leander et al. it was showed that the oldest age group with asthma had a significantly lower physical well-being score than the younger age groups but the social wellbeing was significantly lower among the middle aged. ${ }^{[11,14]}$ In Indian context the middle aged (30-50 years) patients are burdened by the family affairs. The stress due to financial crises and family responsibility has an impact on HRQoL has seen in the present study. In young asthma patients there is comparatively better physical activity and emotional less burdened by the family crises which may be responsible for better HRQoL. The family-centered care for elderly patients may contribute for better HRQOL. The elderly patients are better adapted to the disease condition because of longer exposure that may explain the better HRQoL seen in them.

Previous study by Kalpaklioğlu and Baççioğlu has shown that overweight asthma patients had greater impairment in HRQoL than patients with normal BMI, which is in accordance with the present study. ${ }^{[9]}$ In the present study, the underweight and obese patients had impairment in HRQoL with maximum impairment in the obese group thus BMI contributing to HRQoL. Thus, female, sex, and BMI were found to be the major determinants of impaired quality of life in Asthma patients.

A positive correlation was seen between PFT values and HRQoL scores in the study by P. P. Katz et al. which was confirmed by the present study thus suggesting that the severity of asthma has impact on HRQoL. ${ }^{[15]}$ A similar study by Ziora et al. in children with asthma has shown a positive correlation between PFT and HRQoL scores. ${ }^{[16]}$ Kolawole et al. in their study have shown that lesser the PFT value less is the overall HRQoL confirming that the the most severe the patients diseased condition, the worse the patient's quality of life. ${ }^{[17]}$

Some studies have shown asthma has impact on quality of life but there was no significant correlation between PFT and HRQoL scores. ${ }^{[18]}$ It is most likely that the relationship between HRQoL and asthma severity is due to the relationship between HRQOL and asthma symptoms. Limitation of this study is that assessment was done only once thus change in patients' diseased state and quality of life during subsequent visits was not assessed. Thus assessment of HRQoL would be of value in healthcare research, and eventually, could be used in routine primary care. Additional insights into symptoms and well-being scores will also improve our understanding of how these scales relate to comorbid conditions, and the use of healthcare services. Thus inclusion of health and patient-focused measures of impact in population monitoring for asthma is important for guiding clinical management, predicting health outcomes, formulating clinical policy and assisting in the allocation of resource. 


\begin{tabular}{|c|c|c|c|c|c|}
\hline $\begin{array}{l}\text { PFT } \\
\text { Parameters }\end{array}$ & Symptoms & $\begin{array}{c}\text { Activity } \\
\text { limitation }\end{array}$ & $\begin{array}{l}\text { Emotional } \\
\text { function }\end{array}$ & $\begin{array}{c}\text { Environmental } \\
\text { stimuli }\end{array}$ & Over all \\
\hline$\overline{\Delta F V C}$ & 0.2 & 0.12 & 0.15 & 0.10 & 0.16 \\
\hline$\Delta \mathrm{FEV} 1$ & 0.2 & 0.19 & 0.18 & 0.12 & 0.2 \\
\hline$\Delta \mathrm{FEV} 1 / \mathrm{FVC}$ & 0.07 & 0.14 & 0.12 & 0.06 & 0.11 \\
\hline$\triangle \mathrm{PEF}$ & 0.21 & 0.21 & 0.19 & 0.07 & 0.21 \\
\hline$\triangle$ FEF 25-75/FVC & 0.11 & 0.19 & 0.15 & 0.13 & 0.17 \\
\hline
\end{tabular}

$\mathrm{AQLQ}=$ Asthma Quality of Life Questionnaire, PFT = Pulmonary function tests, FVC = Forced vital capacity, FEV $1=$ Forced expiratory volume in $1 \mathrm{~S}, \mathrm{FEF}=$ Forced expiratory flow, PEF = Peak expiratory flow

\section{ACKNOWLEDGMENT}

The authors like to acknowledge Indian Council of Medical Research (ICMR) for sponsoring the study.

\section{REFERENCES}

1. Masoli M, Fabian D, Holt S, Beasley R, Global Initiative for Asthma (GINA) Program. The global burden of asthma: Executive summary of the GINA Dissemination Committee report. Allergy 2004;59:469-78.

2. Mahapatra P. Social, Economic and Cultural Aspects of Asthma: An Exploratory Study in Andhra Pradesh. Hyderabad: Hyderabad Institute of Health Systems; 1993.

3. Voll-Aanerud M, Eagan TM, Plana E, Omenaas ER, Bakke PS, Svanes C, et al. Respiratory symptoms in adults are related to impaired quality of life, regardless of asthma and COPD: Results from the European community respiratory health survey. Health Qual Life Outcomes 2010;8:107.

4. Juniper EF. Using humanistic health outcomes data in asthma. Pharmacoeconomics 2001;19 (Suppl 2):13-9.

5. Juniper EF, Guyatt GH, Epstein RS, Ferrie PJ, Jaeschke R, Hiller TK. Evaluation of impairment of health related quality of life in asthma: Development of a questionnaire for use in clinical trials. Thorax 1992;47:76-83.

6. Pont LG, van der Molen T, Denig P, van der Werf GT, Haaijer-Ruskamp FM. Relationship between guideline treatment and health-related quality of life in asthma. Eur Respir J 2004;23:718-22.

7. Omachi TA. Poor outcomes and asthma hospitalisations: How important is asthma severity and how do we measure it? Allergol Immunopathol (Madr). 2009;37:223-4.

8. Juniper EF, Buist AS, Cox FM, Ferrie PJ, King DR. Validation of a standardized version of the Asthma Quality of Life Questionnaire. Chest 1999;115:1265-70.

9. Kalpaklioğlu AF, Baççioğlu A. Evaluation of quality of life: Impact of allergic rhinitis on asthma. J Investig Allergol Clin Immunol 2008;18:168-73.
10. Dougherty RH, Fahy JV. Acute exacerbations of asthma: Epidemiology, biology and the exacerbation-prone phenotype. Clin Exp Allergy 2009;39:193-202.

11. Leander M, Lampa E, Janson C, Svärdsudd K, Uddenfeldt M, RaskAndersen A. Determinants for a low health-related quality of life in asthmatics. Ups J Med Sci 2012;117:57-66.

12. Skrzypulec V, Drosdzol A, Nowosielski K. The influence of bronchial asthma on the quality of life and sexual functioning of women. J Physiol Pharmacol 2007;58 Suppl 5:647-55.

13. Naleway AL, Vollmer WM, Frazier EA, O'Connor E, Magid DJ. Gender differences in asthma management and quality of life. J Asthma 2006;43:549-52.

14. Leander $M$, Janson $C$, Uddenfeldt M, Cronqvist A, Rask-Andersen A. Associations between mortality, asthma, and health-related quality of life in an elderly cohort of Swedes. J Asthma 2010;47:627-32.

15. Katz PP, Eisner MD, Henke J, Shiboski S, Yelin EH, Blanc PD. The Marks Asthma Quality Of Life Questionnaire: Further validation and examination of responsiveness to change. J Clin Epidemiol 1999;52:667-75.

16. Ziora D, Madaj A, Wieckowka E, Ziora K, Kozielski K. Correlation of spirometric parameters taken at a single examination with the quality of life in children with stable asthma. J Physiol Pharmacol 2007;58 Suppl 5:801-9.

17. Kolawole MS, Olayemi AF, Gregory EE, Abiodun AO, Daniel OO, Bamidele $A O$. Health related quality of life and psychological variables among a sample of asthmatics in Ile-Ife South-Western Nigeria. Libyan J Med 2011;6.

18. Rutishauser C, Sawyer SM, Bowes G. Quality-of-life assessment in children and adolescents with asthma. Eur Respir J 1998;12:486-94.

How to cite this article: Nalina N, Sathish Chandra M R, Umashankar. Assessment of quality of life in bronchial asthma patients. Int J Med Public Health 2015;5:93-7.

Source of Support: Indian Council of Medical Research, Conflict of Interest: None declared. 